\title{
Évaluation de la durabilité des exploitations agricoles irriguées en zones arides tunisiennes par la méthode IDEA: cas de la région de Zarzis
}

\author{
Mohamed Amir Bouzaida*, Houda Doukali*
}

DOI: $10.30682 / \mathrm{nm} 1904 \mathrm{~g}$

JEL codes: Q01, Q15, Q25

\begin{abstract}
In southern Tunisia water for irrigation is very important. Its management requires adequate control to ensure the sustainability of the irrigated lands. Affected by desertification, natural resources are limited. Intensification and excessive irrigation are leading to the degradation of the quality of these resources, imposing an obstacle for agricultural development.

In this work, an analytical study of irrigated perimeters in Zarzis was carried out to identify their main characteristics. The aim is to evaluate the socio-economic and environmental impacts of irrigation water on farming system sustainability, using the IDEA method by analyzing the three sustainability scales (agro-ecological, socio-territorial and economic). The study is based on a sample of 39 farms, characterized by a limited socio-territorial scale, in which the component "Quality of products and territories" is unsatisfactory. The economical aspect it is characterized by a good transmissibility, depending on financial autonomy degree. The results in the agro-ecological scale are the highest. The assessment of the sustainability of irrigated lands requires the analysis of interactions between these three scales.
\end{abstract}

Keywords: Zarzis, Irrigation, Desertification, Sustainability, Evaluation, Impact, IDEA.

\section{Introduction}

La Tunisie, en raison de sa situation géographique, est un pays aride sur la majeure partie de son territoire. Cette aridité, conjuguée à la variabilité du climat méditerranéen, fait de l'eau une ressource à la fois rare et inégalement répartie dans le temps et dans l'espace. Cette ressource est de plus en plus sollicitée avec l'apparition de graves phénomènes de surexploitation des nappes, entraînant une salinisation des eaux et une détérioration des terres agricoles, particu- lièrement dans les périmètres irrigués avec des eaux chargées en sel.

A cette aridité climatique, caractérisée par la rareté et l'irrégularité de la pluie et l'agressivité des averses et des vents violents, s'ajoute le mode d'exploitation inapproprié des ressources naturelles, étant à l'origine de la vulnérabilité du couvert végétal naturel et des sols. Les pratiques culturales ne font qu'amplifier le phénomène de la désertification, surtout au Sud tunisien.

Pour optimiser et rationaliser la gestion ainsi que l'exploitation des ressources natur-

\footnotetext{
* Institut des Regions Arides, Médenine, Tunisia.

Corresponding author: amir.bouzaida@gmail.com
} 
elles, l'Etat est intervenu à travers nombreux programmes de mise en valeur, allant de pair avec des actions de développement social. Ces processus de développement font appel à des niveaux d'intensification élevés et à des techniques modernes (Sghaier et al., 2000). Ces actions ont été réalisées donc, pour une meilleure gestion des ressources naturelles, mieux lutter contre la désertification et la préservation des ressources naturelles.

Cette intensification est traduite entre autres par une forte exploitation des ressources hydriques par l'installation des périmètres irrigués, ayant entrainé une détérioration de la qualité de ces eaux et une augmentation de la salinité par l'utilisation à grande échelle des eaux relativement ou fortement salées en irrigation.

Ceci a affecté non seulement le niveau des rendements des cultures, mais aussi a provoqué la dégradation à long terme et souvent irréversiblement, de la structure des sols. Ces problèmes risquent de compromettre la durabilité des périmètres en question, surtout avec l'exploitation excessive et inappropriée des ressources naturelles, accentuant davantage le phénomène de désertification.

Aussi, il faut souligner que les programmes de vulgarisation et de formation agricole ne sont pas adaptés aux besoins des exploitations et n'intègrent pas les notions de durabilité dans la préservation des ressources naturelles. Cette situation a amplifié l'exode rural des jeunes et l'augmentation du taux de chômage dans les régions à vocation agricole (Ferchichi et al., 2015).

Dans ce cadre, la mise en valeur intensive sous irrigation dans les zones semi-arides et arides conduit le plus souvent à la dégradation de la qualité des ressources en sol et en eau. L'ampleur de la dégradation est fortement liée à la qualité de l'eau d'irrigation, à la non maîtrise de la trilogie : Irrigation-Salinité-Drainage et aux pratiques de mise en valeur agricole non rationnelles (Debbarh et al., 2007). En outre, une gestion économe et autonome des exploitations agricoles garantit une efficacité économique et des revenus décents aux agriculteurs. Elle permet une baisse des charges, une diminution des coûts pour la collectivité tout en valorisant le travail. Elle se fonde sur une équité sociale qui permet de valoriser le travail et les emplois avec un partage équitable des richesses tout en respectant les normes de durabilité agricole.

La région aride de Zarzis se trouve dans cette dynamique, avec une salinisation des périmètres irrigués qui vient s'ajouter à une érosion éolienne, accentuant, ainsi, une désertification de plus en plus accrue et menaçante pour la production agricole.

\section{Evaluation de la durabilité des exploita- tions}

L'objectif global de cette étude est de mesurer la durabilité des exploitations agricoles dans les périmètres irrigués dans cette zone aride tunisienne et identifier les impacts socio-économiques et environnementaux de cette dynamique de transition territoriale par la méthode des indicateurs de durabilité des exploitations agricoles (IDEA). Nonobstant, avant le recours à ladite technique, un diagnostic technique et socio-économique des périmètres irrigué est accompli. Les données collectées seront analysées et interprétées pour comprendre le fonctionnement des ces périmètres. Les étapes d'évaluation de la durabilité sont comme suit :

\subsection{Diagnostic des exploitations}

Le diagnostic d'un périmètre irrigué est une sorte d'analyse qui permet d'identifier les causes $\mathrm{du}$ dysfonctionnement d'un système d'irrigation et proposer des solutions et actions contre les principales contraintes qui entravent les performances du périmètre irrigué sur le plan technique, social, économique et environnemental en identifiant les principaux facteurs limitant et les opportunités pour améliorer la productivité et la durabilité d'un système agricole irrigué.

Ce diagnostic assure ainsi, une description des principales caractéristiques des systèmes agricoles irrigués pour pouvoir effectuer une évaluation de fonctionnement du système irrigué. Selon (Bonneviale et al., 1989), " un diagnostic d'exploitation est le résultat d'une démarche d'investigation visant à identifier et à apprécier les forces et les faiblesses de celle-ci et à en rechercher les causes. Le diagnostic est toujours 
relié au point de vue et au référentiel de celui qui le fait ". C'est alors, un jugement porté d'une situation à partir de l'analyse d'indicateurs ou de paramètres. Il s'agit de déterminer les causes des problèmes existants sur le périmètre irrigué et de proposer des solutions d'amélioration.

Le diagnostic escompté doit être dynamique et permettrait de comprendre l'évolution du système irrigué et d'analyser la diversité des situations et les processus de différenciation à l'intérieur du système irrigué (Apollin et al., 2012).

\subsection{Evaluation des impacts de l'installation des périmètres irrigués}

Une évaluation d'impact est indispensable pour mesurer la durabilité des exploitations agricoles. Elle se traduit par la mise en œuvre de démarches et outils qui reposent sur le choix de critères et d'indicateurs de durabilité choisis à priori, ainsi que sur des méthodes de calcul, de pondération et d'agrégation aboutissant à attribuer des notes synthétiques de performance (Landais, 1998).

Dans la plupart des méthodes, les critères de l'évaluation à l'échelle de l'exploitation agricole, se sont progressivement conformes aux trois dimensions constitutives de la durabilité: être durable c'est économiquement viable, so- cialement équitable et écologiquement reproductible. (Barbier et al., 2010).

En agriculture, ce triptyque a parfois évolué vers une version à quatre dimensions: viabilité, vivabilité, reproductibilité et transmissibilité. Selon (Feschet et al., 2014) plusieurs méthodes françaises d'évaluation multicritère appliquées aux systèmes agricoles ont fait l'objet d'une analyse approfondie. Le choix s'est fondé sur deux paramètres :

- Les dimensions du développement durable considérées, afin de couvrir les aspects sociaux et économiques

- La reconnaissance par les acteurs du monde agricole, pour garantir leur légitimité.

\section{Matériel et méthodes}

\subsection{La zone d'étude}

La région de Zarzis est une presque ile du Gouvernorat de Médenine, s'étendant sur 340 $\mathrm{Km}^{2}$. son emplacement lui accorde un caractère stratégique comme l'une des zones économiques les plus diversifiés au Sud tunisien (agriculture, pêche, industrie, tourisme...). Elle est segmentée en 16 unités sectorielles qui dégagent une forte diversité selon la superficie et la population relative chaque unité (Figure 1).

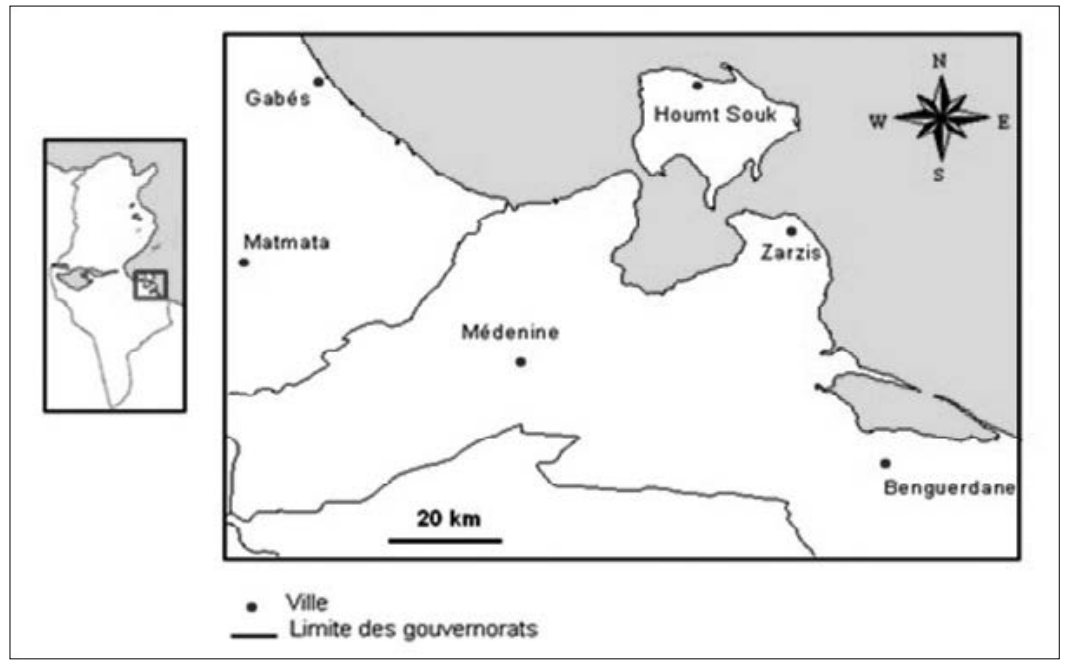

Figure 1. Carte de localisation de la région de Zarzis. 
La zone d'étude se caractérise par une pluviométrie faible, irrégulière et sporadique, des températures élevées et un bilan hydrique déficitaire durant toute l'année. Elle est considérée parmi les régions les plus touchées par la dégradation de ses ressources agricoles. Ceci a poussé sa population à l'émigration, principalement vers l'étranger. A cet égard, l'Etat a essayé d'encourager l'extension des activités agricoles permettant ainsi, de contribuer à l'amélioration des indicateurs de développement de la région et par conséquent, à atteindre un développement durable par le biais du secteur agricole, en général, et les périmètres irrigués en particulier.

\subsection{Echantillonnage}

Le recueil des données nécessaires a été effectué dans la zone d'étude durant la période Aout-novembre 2016. Un premier critère de choix des exploitants était leur appartenance à la liste officielle du ministère des agriculteurs se trouvant dans les périmètres irrigués (CRDA Médenine). L'échantillon était au nombre de 39, répartis sur tous les secteurs de la région (Figure 2), pour une meilleure représentativité. Les données recueillies avaient pour objectif d'avoir des éléments d'identification qualitatifs et quantitatifs de la situation générale de leurs respectives exploitations agricoles.

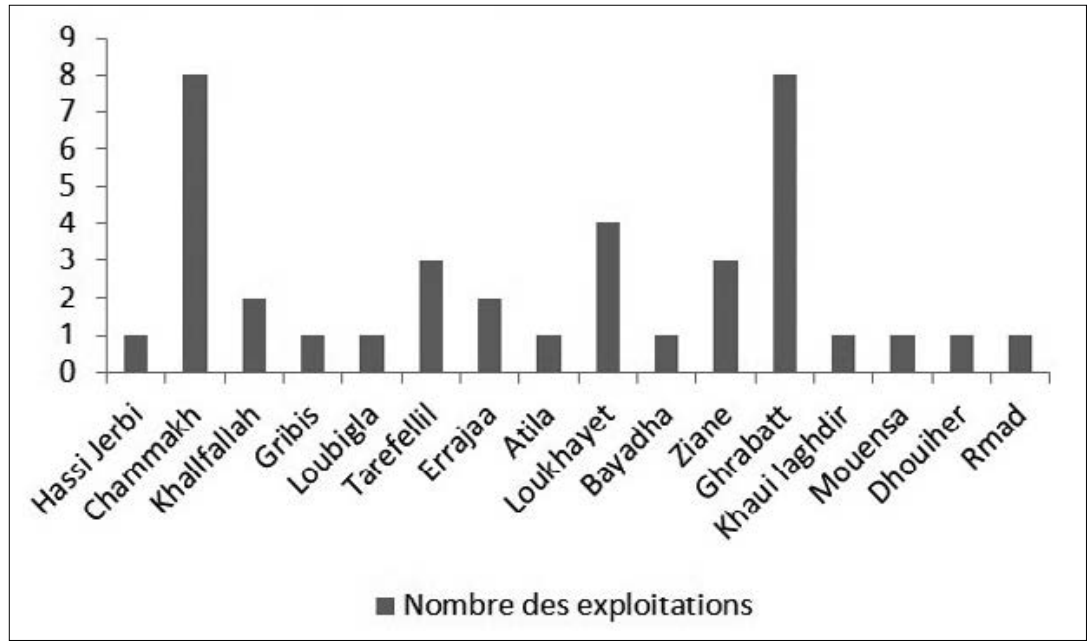

Figure 2 - La répartition des exploitations selon les secteurs.

\subsection{Questionnaire}

Pour atteindre cet objectif une étude descriptive a été réalisée pour dégager les différentes caractéristiques des exploitations agricoles en se basant sur un diagnostic du fonctionnement (sur les plans technique, socio-économique et environnemental). Pour cela, un travail de terrain a été nécessaire pour complémenter ces informations quantitatives obtenues par une information qualitative à travers des réunions, entretiens et des enquêtes dans la zone d'étude. Pour le travail de terrain, il était nécessaire d'élaborer un questionnaire regroupant un ensemble de questions concernant l'agriculteur, la structure de l'exploitation, les équipements de production, le système cultural, les données technico-économiques d'exploitation et les rapports de l'exploitation avec son environnement économique et institutionnel. On s'intéresse essentiellement à la gestion des cultures irriguées et à l'usage de l'eau d'irrigation selon la nature de la ressource. Ceci nous permettra de cerner les différentes pratiques et les adaptations stratégiques des irrigants. 


\subsection{Méthode de travail}

\subsubsection{Justification du choix de la méthode}

Le choix est axé sur la méthode IDEA(Version 3), car il s'agit d'un essai de quantification de la durabilité. Elle analyse les forces et les faiblesses du système de production au niveau de l'exploitation. De plus, les indicateurs cités dans la méthode caractérisent les concepts-clés issus de la définition d'une agriculture durable. C'est un outil d'évaluation de la durabilité des systèmes agricoles qui repose sur une évaluation quantitative des pratiques jugées favorables au milieu biophysique et social. Les itinéraires techniques (assolement, rotations, fertilisations...) et les pratiques sociales et territoriales de la production sont ainsi, considérés comme une « unité de durabilité » positives ou négatives et proportionnelles aux impacts sur les différentes caractéristiques environnementales et sociales du milieu (Vilain, 2003).

\subsubsection{Principes et mode de calcul de la méthode IDEA}

Chaque composante regroupe plusieurs indicateurs, soit au total 41 indicateurs de durabilité, eux-mêmes constitués d'un ou plusieurs items élémentaires, caractérisant une pratique (ou une caractéristique) et contribuant à la valeur finale de l'indicateur. Les objectifs de l'échelle agroécologique se réfèrent aux principes agronomiques de l'agriculture intégrée (Viaux, 1999).

Ils doivent permettre l'efficacité économique pour un coût écologique aussi faible que possible. Ceux de l'échelle de durabilité socio-territoriale se réfèrent davantage à l'éthique et au développement humain, caractéristiques essentielles des systèmes agricoles durables. Enfin, les objectifs de l'échelle de durabilité économique précisent les notions essentielles liées à la fonction entrepreneuriale de l'exploitation.

Le nombre de points ou d'unités de durabilité attribués à chaque indicateur est donc, compris entre les bornes zéro (même si la somme des items élémentaires est négative) et une valeur plafond qui est propre à chaque indicateur (même si la somme de ses items élémentaires est supérieure). Chaque composante est également limitée, de la même manière, à une valeur plafond (généralement 33 points) qui pondère son poids relatif et autorise un très grand nombre de combinaisons techniques pour l'atteindre.

Le mode de calcul, il est basé sur un système de points avec un plafonnement. Les trois échelles de durabilité sont du même poids et varient de 0 à 100 points. L'ensemble des informations est traduit en unités élémentaires de durabilité déterminant la note attribuée à chaque indicateur.

Le score d'une exploitation pour chacune des trois échelles de durabilité est le nombre cumulé d'unités élémentaires de durabilité (ou de points) obtenues pour divers indicateurs de l'échelle considérée. Plus la note est élevée, plus l'exploitation est considérée comme durable pour l'échelle considérée.

Ce mode de calcul autorise sur l'exploitation agricole un très grand nombre de combinaisons techniques pour atteindre un même degré de durabilité. En effet, même si certains principes sont communs à tous les systèmes agricoles durables, nous considérons qu'il n'y a pas de modèle unique. La diversité des contextes et des milieux de production et la diversité des systèmes de production et des combinaisons techniques autorisent de très nombreux chemins pour progresser. Certaines faiblesses techniques ou structurelles peuvent donc être partiellement compensées par des options plus compatibles avec l'organisation générale du système de production. (Abdenour, 2008).

\subsubsection{Analyse empirique}

Sur la base des données collectées, une analyse empirique a été effectuée en vue d'évaluer la durabilité des exploitations irriguées faisant l'objet de notre étude. On décrira les résultats de l'étude, on calculera l'indicateur utilisé et on indiquera la note attribuée par la méthode IDEA pour chaque échelle. Successivement, on procédera à une appréciation de la durabilité globale des exploitations. 


\section{Résultats et discussions}

Dans ce qui suit, on mettra en avant les résultats de l'étude technico-économique du fonctionnement des ces périmètres, et en second lieu, on va mesurer leur degré de durabilité à l'aide d'une approche d'évaluation des impacts IDEA.

\subsection{Analyse foncière}

\subsubsection{La répartition des exploitations selon la taille}

Sur une superficie totale de l'ordre de $311 \mathrm{Ha}$, $60 \%$ de ladite superficie est conduit en mode irrigué. Les lots étudiés ont des tailles variant entre 0,4 et $43 \mathrm{Ha}$; avec une superficie moyenne attribuées de l'ordre de 4,8 Ha/exploitant, ce qui est une superficie très limitée, ayant généralement une vocation familiale.

L'extension des superficies irriguées est assez difficiles à cause de la salinité de l'eau d'irrigation (Dhouiher, khaouilaghdir, Hassi jerbi), des problèmes de désertification et ensablement (Loubigla, Loukhayet, Bayadha, Ghrabat), du manque de la main d'œuvre (Rmad,chmmakh) et d'autres facteurs anthropiques comme l'urbanisation (Atila, Erraja, Gribis et Mouensa).

\subsubsection{Principales spéculations agricoles}

Concernant l'occupation du sol, l'olivier est la culture dominante dans ces exploitations (Figures 3 et 4).

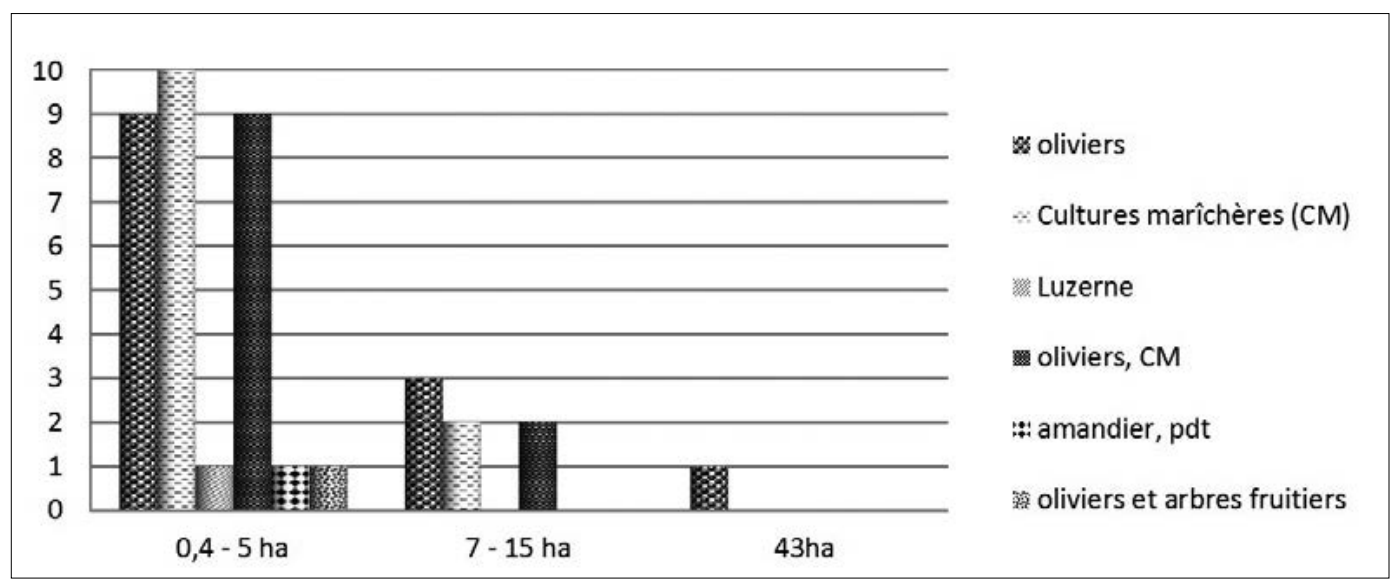

Figure 3. Répartition selon l'occupation principale.

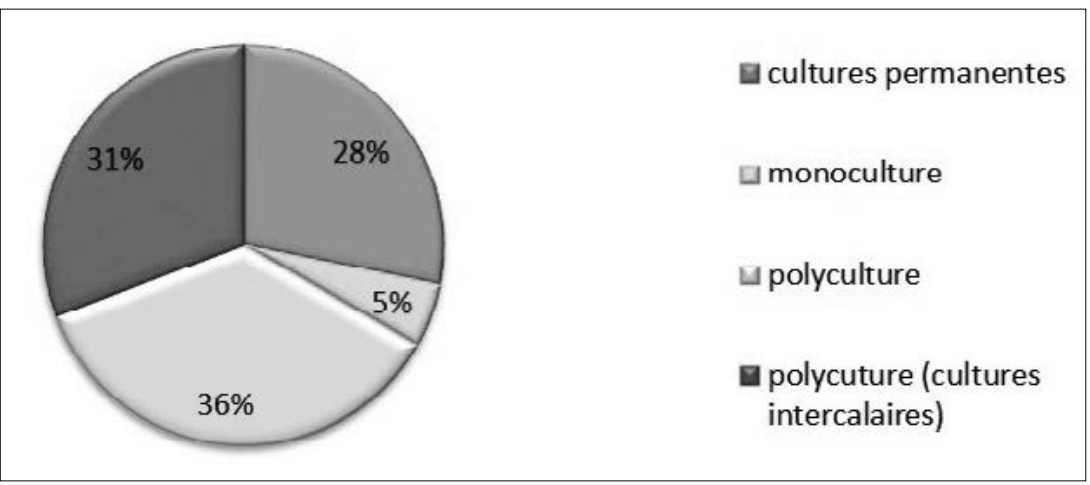

Figure 4. Le système de culture. 


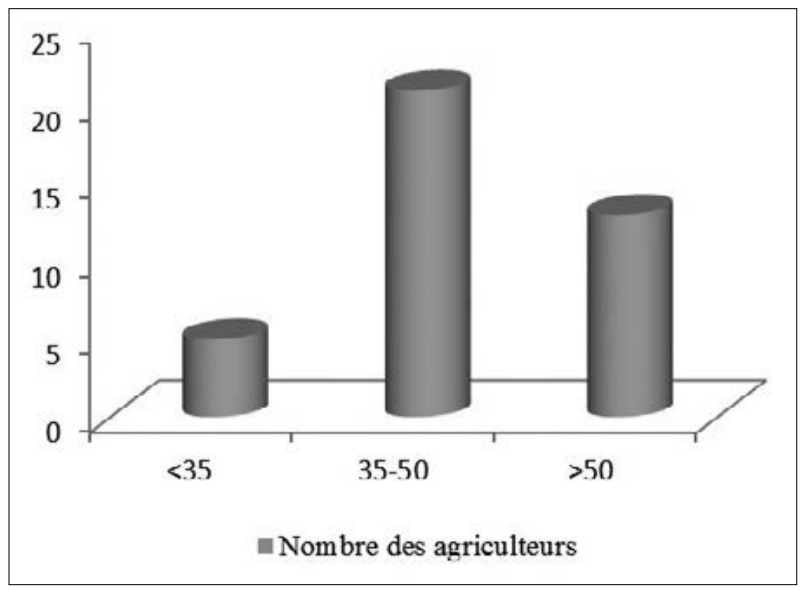

Figure 5 - Répartition des agriculteurs selon l'âge.

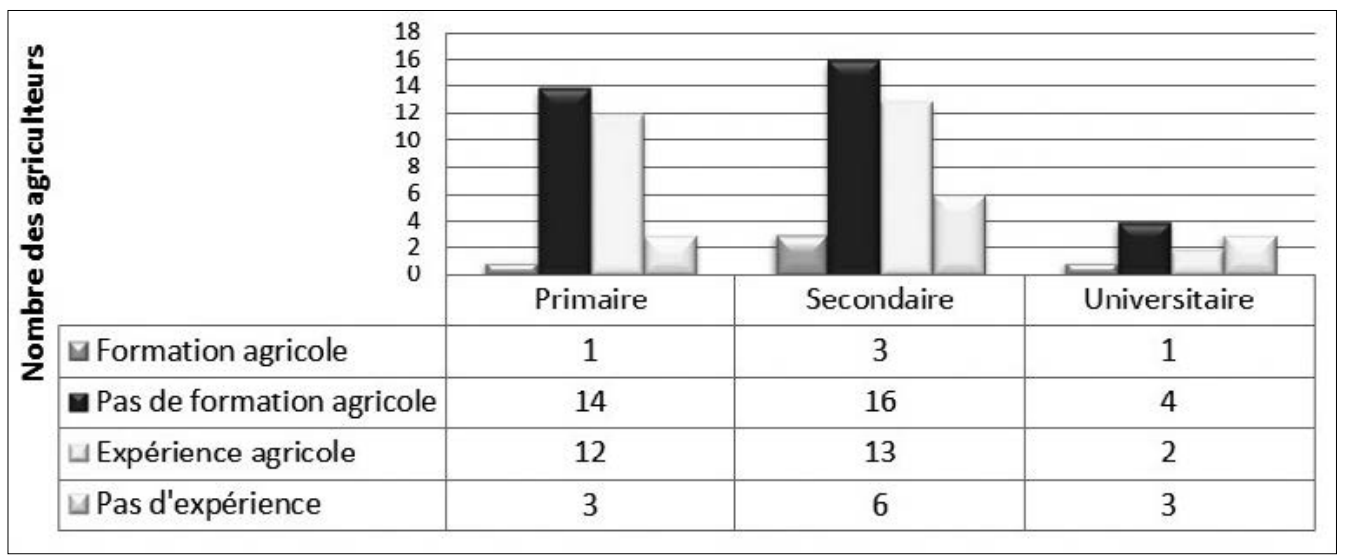

Figure 6 - Répartition des agriculteurs selon le niveau de formation.

Les exploitations de petites tailles 0-5 ha sont les plus diversifiées, avec un système de polyculture et des cultures intercalaires. Les cultures agricoles sont intensifiées dans le temps et dans l'espace. Les agriculteurs entretiennent plus d'une culture sur la même parcelle pour augmenter la productivité, éviter le gaspillage de l'eau et réduire les coûts.

\subsubsection{La répartition des agriculteurs selon l'âge}

La moyenne d'âge des exploitants est de 47 ans, oscillant entre 20 et 70 ans (Figure 5).

Il faut signaler que $54 \%$ des agriculteurs ont un âge compris entre 35 et 50 ans, avec peu de présence des jeunes agriculteurs (seulement $13 \%$ du nombre total des agriculteurs). Le vieil- lissement des agriculteurs reflète une alarmante absence de relève générationnelle, étant dû au manque d'intérêt des jeunes à travailler dans le secteur primaire. Ces jeunes sont beaucoup plus attirés par d'autres activités comme les services ou le tourisme ; ou bien ils sont tentés par l'immigration vers l'Europe. Un peu moins de 50\% des jeunes émigrants vers le continent européen du Gouvernorat de Médenine proviennent de la Délégation de Zarzis entre les années 2009 et 2014 (ODS, 2015).

\subsubsection{La répartition des agriculteurs selon la formation}

La Figure 6, ci-dessous, regroupe les agriculteurs selon leur niveau d'instruction ou leur formation agricole. 
Le niveau d'instruction des exploitants est hétérogène. $13 \%$ ont un niveau supérieur, $49 \%$ ont un niveau secondaire et $38 \%$ ont un niveau primaire. Quant à la formation agricole, seulement $13 \%$ des agriculteurs ont reçu ce type de formation par les institutions spécialisées (CTV, AVFA, APIA, UTAP, Centres de formation agricole ou de recherche...).

\subsection{Analyse technique des périmètres irrigués}

\subsubsection{Resources en eau}

Le déficit des précipitations a entraîné le recours à l'eau de surface par la mise en place de puits et forages privés. Dans la zone d'étude on note l'existence de 51 puits au niveau de cette nappe. Il se répartissant en 9 puits équipés des groupes motopompes (un moteur à essence ou à gasoil), 30 puits utilisés par des groupes électropompes (avec un moteur électrique) et 12 puits abandonnés.

Cette abondance des puits est due principalement à plusieurs raisons, telles que: la dégradation continue de la qualité et la quantité d'eau disponible, l'usage accru de l'eau d'irrigation, l'installation de nouveaux périmètres irrigués, l'extension de l'urbanisation, mais surtout l'absence de contrôle des services étatiques, concernés de ces nouveaux forages, faisant fi des législations très restrictives en vigueur. Les profondeurs des puits sont variables, allant de 90 à
$110 \mathrm{~m}$, avec un degré de salinité élevé, qui se situe entre 2 et $7 \mathrm{~g} / 1$.

On remarque aussi, l'existence de 30 réservoirs d'eau pour accroître les réserves hydrauliques. Il s'agit de réservoirs peu profonds avec des capacités qui varient de 5 à $60 \mathrm{~m}^{3}$ et qui ne retiennent généralement de l'eau que pendant une courte période de l'année.

\subsubsection{Techniques d'irrigation}

Le système d'irrigation dominant est basé principalement sur la micro-irrigation, permettant une meilleure optimisation de la gestion de l'eau. Il s'agit de micro-asperseurs ainsi que des systèmes de goutte à goutte. D'autres agriculteurs utilisent la technique de submersion (Figure 7).

La répartition des techniques d'irrigation dans les exploitations est comme suit :

- $56 \%$ utilisant la technique de goutte à goutte ;

- $8 \%$ adoptant la technique de goutte à goutte en complémentarité avec l'aspersion ;

- $15 \%$ des irrigants utilisent la technique de goutte à goutte en ajoutant de la submersion ;

- $21 \%$ des exploitants utilisent la submersion.

Les principales difficultés des agriculteurs dans cet aspect technique sont relatives aux coûts initiaux, considérés très élevés, et les risques de bouchage du système, tout particulièrement les distributeurs. Une bonne conception, une gestion rationnelle et une maintenance régulière peuvent éviter ces problèmes.

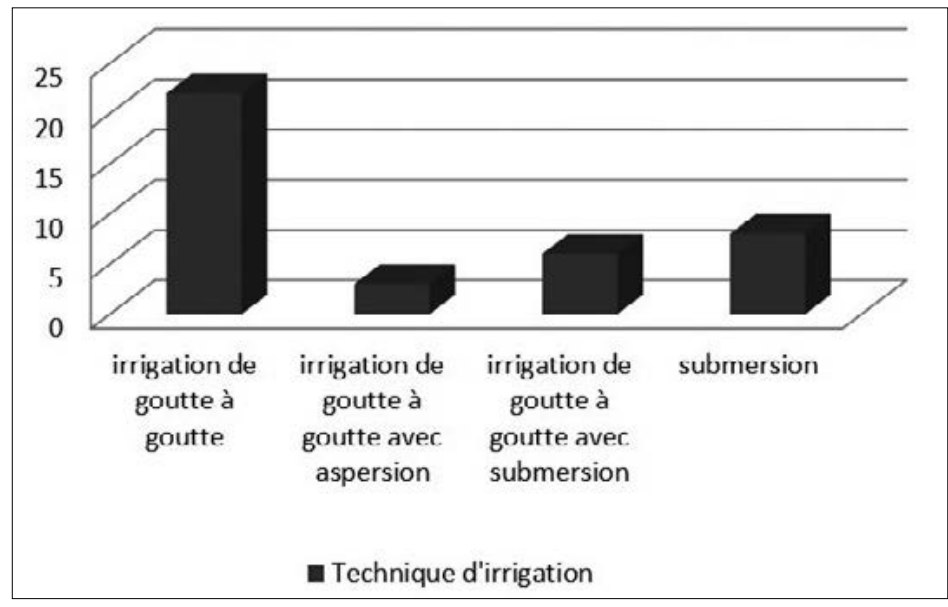

Figure 7 - Les techniques d'irrigation. 


\subsubsection{Mécanisation}

En jouant un rôle fondamental dans la plupart des exploitations, la mécanisation intervient dans la préparation du sol, la fertilisation, la protection phytosanitaire, la récolte, la taille, etc. Dans ce sens, $87 \%$ des agriculteurs font le labour par des tracteurs, $8 \%$ utilisent la traction animale et $5 \%$ n'ont pas recours à la mécanisation.

\subsection{Analyse économique et estimation de la rentabilité}

Dans l'aspect économique on a calculé les marges brutes des exploitations enquêtées pour avoir une idée sur leurs respectives rentabilités. En guise de résultat, on a découvert qu'il y a plusieurs niveaux de marges brutes qui se diffèrent d'un exploitant à un autre. On peut les agrouper en deux groupes :

- Groupe 1 : formé par 10 exploitations qui ont une marge brute négative. Ceci est dû à plusieurs raisons, telles que: l'irrégularité des rendements, le manque de savoir-faire agricole, et la gestion inappropriée des ressources (hydriques, humaines, matérielles...). Dans ce groupe, il y a un problème de rentabilité, voire logiquement de viabilité des exploitations concernées. Beaucoup de ces structures agricoles ont été délaissés et abandonnées, non seulement faute de rentabilité claire montrée ci-haut, mais aussi par manque d'intérêt ou à cause d'une forte salinité de l'eau d'irrigation.

- Groupe 2 : formé par 29 exploitations qui ont une marge brute positive. Cette marge varie entre 33 et $13966 \mathrm{DT} / \mathrm{Ha}$. Ceci est grâce à l'intensification du système oléicole en irrigué, des différentes techniques utilisées et du volume d'investissement important, surtout de la part des propriétaires ayant d'autres activités extra-agricoles ou résidant à l'étranger.

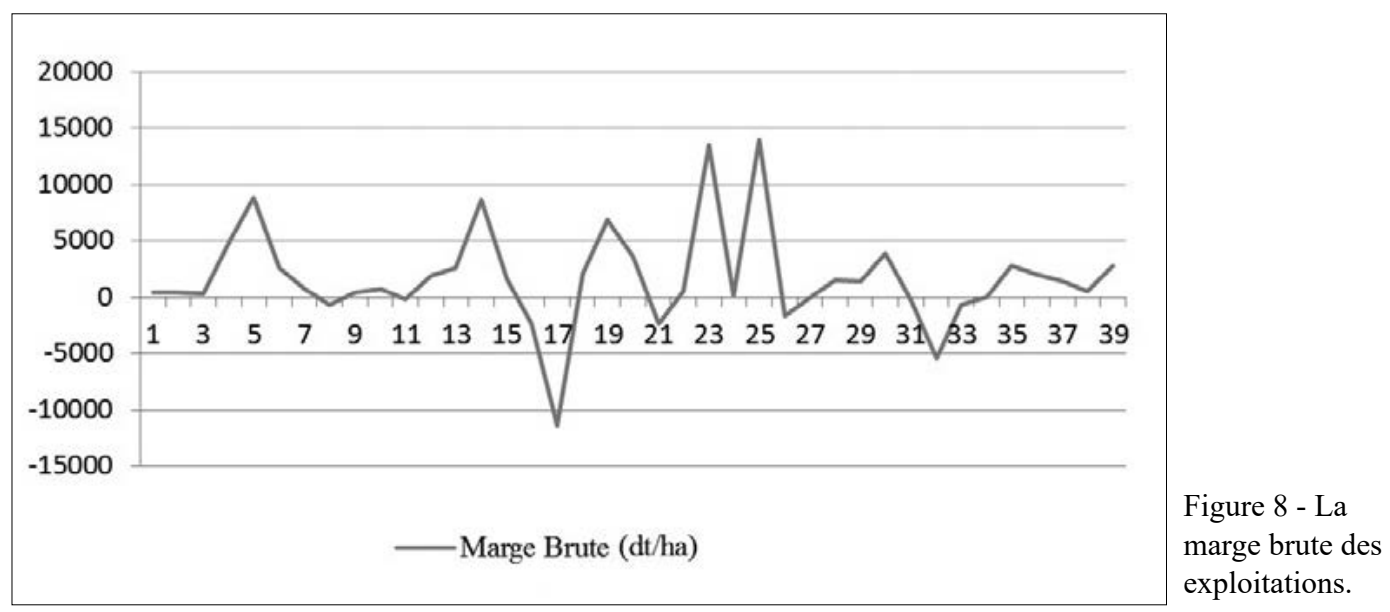

\subsection{Analyse des résultats de la méthode IDEA}

\subsubsection{Résultat global}

Les résultats obtenus (Figure 9) ont montré que la durabilité des exploitations en périmètre irrigué est limitée par l'échelle socio-territoriale avec une note de 48/100. A l'opposé, l'échelle agro-écologique présente une note de 70/100, alors que l'échelle économique est de l'ordre de $64 / 100$.

Selon la représentation graphique de ces composante de Durabilité, on remarque que les indicateurs viabilité, organisation de l'espace et efficience du processus productif ont les valeurs les plus faibles, avec respectivement $7 ; 8$ et 12 . 


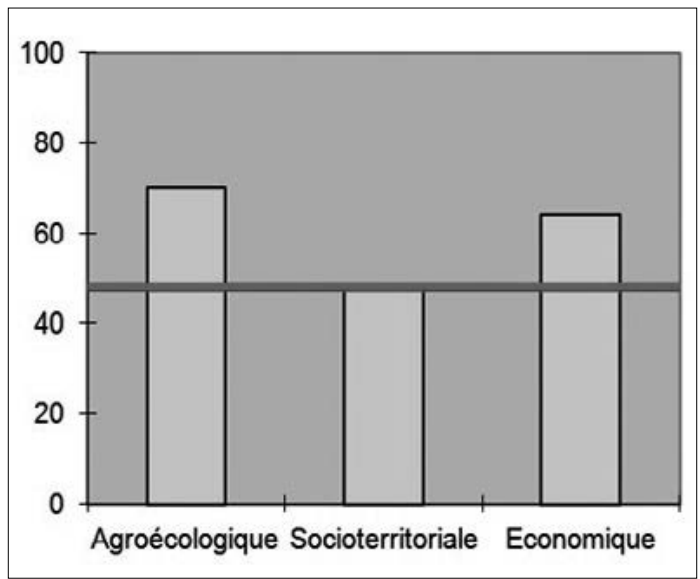

Figure 9 - Graphique de la Durabilité des exploitations.

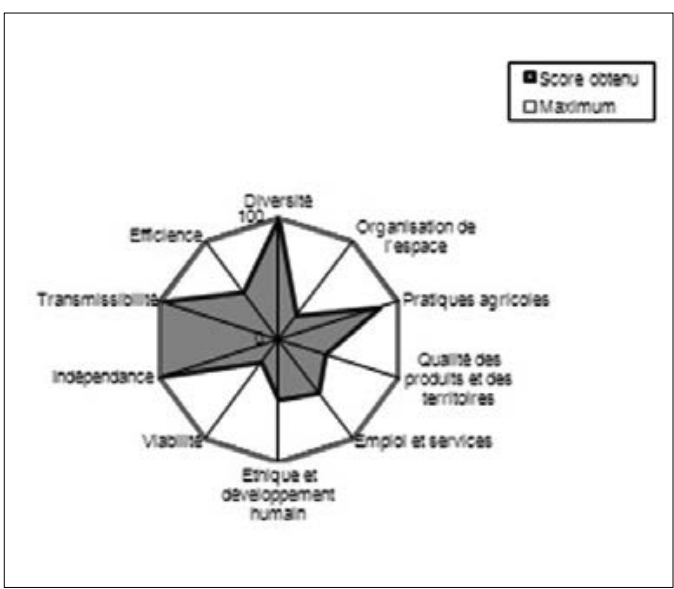

Figure 10 - Représentation graphique des composantes de Durabilité des exploitations.

Tableau 1 - Notes de l'échelle de durabilité agro-écologique.

\begin{tabular}{|c|c|c|c|}
\hline Composante & Indicateurs & Score & Intervalle \\
\hline \multirow{4}{*}{$\begin{array}{l}\text { Diversité } \\
\text { domestique }\end{array}$} & Diversité des cultures annuelles & 12 & $0-12$ \\
\hline & Diversité des cultures pérennes & 12 & $0-12$ \\
\hline & Diversité animale & 6 & $0-6$ \\
\hline & Valorisation du patrimoine génétique & 3 & $0-3$ \\
\hline \multirow{7}{*}{$\begin{array}{l}\text { Organisation de } \\
\text { l'espace }\end{array}$} & Assolement & 0 & $0-8$ \\
\hline & Dimension des parcelles & 3 & $0-6$ \\
\hline & Gestion de la matière organique & 5 & $0-5$ \\
\hline & Régulation écologique & 0 & $0-12$ \\
\hline & Contribution aux enjeux env. du territoire & 0 & $0-4$ \\
\hline & Valorisation de l'espace & 0 & $0-5$ \\
\hline & Surface fourragères & 0 & $0-5$ \\
\hline \multirow{7}{*}{$\begin{array}{l}\text { Pratiques } \\
\text { agricoles }\end{array}$} & Fertilisation & 0 & $0-8$ \\
\hline & Effluents organiques liquides & 3 & $0-3$ \\
\hline & Pesticides & 12 & $0-13$ \\
\hline & Traitement vétérinaires & 3 & $0-3$ \\
\hline & Protections des sols & 0 & $0-5$ \\
\hline & Gestion de l'eau & 3 & $0-4$ \\
\hline & Dépendance énergétique & 8 & $0-10$ \\
\hline \multicolumn{2}{|l|}{ Total } & 70 & $0-100$ \\
\hline
\end{tabular}


Les tableaux 1, 2 et 3 illustrent les composantes de chaque échelle et les comparent au maximum. L'analyse de l'échelle agroécologique (Tableau 1) montre bien que pour la composante organisation de l'espace, les indicateurs : Assolement, régulation écologique, contribution aux enjeux environnementaux du territoire et la surface fourragère, ont des notes nulles. Pour l'échelle socio-territoriale (Tableau 2), l'indicateur du travail collectif est nul, ce qui peut être expliqué par le manque de structures coopératives entre les agriculteurs. L'étude de l'échelle économique (Tableau 3) montre que l'indicateur du taux de spécialisation a la valeur la plus faible, avec un score de 2 , ce qui reflète la non-spécialisation des exploitations étudiées.

Tableau 2 - Note de l'échelle de durabilité socio-territoriale.

\begin{tabular}{|c|c|c|c|}
\hline Composante & Indicateurs & Score & Intervalle \\
\hline \multirow{5}{*}{$\begin{array}{l}\text { Qualité des } \\
\text { produits et des } \\
\text { territoires }\end{array}$} & Démarche de qualité & 0 & $0-10$ \\
\hline & Patrimoine bâtiment et paysage & 3 & $0-8$ \\
\hline & Gestion des déchets non organiques & 2 & $0-5$ \\
\hline & Accessibilité de l'espace & 3 & $0-5$ \\
\hline & Implication sociale & 5 & $0-6$ \\
\hline \multirow{6}{*}{$\begin{array}{l}\text { Emploi et } \\
\text { services }\end{array}$} & Valorisation des filières courtes & 7 & $0-7$ \\
\hline & Autonomie et valorisation des ressources locales & 2 & $0-10$ \\
\hline & Services et pluriactivités & 2 & $0-5$ \\
\hline & Contribution à l'emploi & 6 & $0-6$ \\
\hline & Travail collectif & 0 & $0-5$ \\
\hline & Pérennité probable & 1 & $0-3$ \\
\hline \multirow{7}{*}{$\begin{array}{l}\text { Ethique et } \\
\text { développement } \\
\text { humain }\end{array}$} & Contribution à l'équilibre alimentaire & 0 & $0-10$ \\
\hline & Qualité du Bienêtre & 3 & $0-3$ \\
\hline & Formation & 1 & $0-6$ \\
\hline & Intensité de travail & 4 & $0-7$ \\
\hline & Qualité de vie & 5 & $0-6$ \\
\hline & Isolement & 3 & $0-3$ \\
\hline & Accueil hygiène et sécurité & 1 & $0-4$ \\
\hline \multicolumn{2}{|l|}{ Total } & 48 & 100 \\
\hline
\end{tabular}

Tableau 3 - Note de l'échelle de durabilité économique.

\begin{tabular}{|l|l|c|c|}
\hline \multirow{2}{*}{ Composante } & Indicateurs & Score & Intervalle \\
\hline \multirow{2}{*}{ Indépilité } & Viabilité économique & 5 & $0-20$ \\
\cline { 2 - 4 } & Taux de spécialisation économique & 2 & $0-10$ \\
\hline Transmissibilité & Autonomie financière & 15 & $0-15$ \\
\cline { 2 - 4 } & Sensibilité aux aides & 10 & $0-10$ \\
\hline Efficience & Efficience du processus productif & 20 & $0-20$ \\
\hline Total & & 12 & $0-25$ \\
\hline
\end{tabular}




\subsubsection{Discussion}

On procédera dans ce volet de discussion à l'explication des scores relatifs à chaque échelle de durabilité, ainsi que leurs respectives composantes pour plus de compréhension.

\section{a) Echelle socio-territoriale}

Cette échelle de durabilité reflète l'insertion de l'exploitation dans son territoire et dans la société. Elle cherche à évaluer la qualité de vie de l'agriculteur et le poids des services qu'il rend au territoire et à la société. En ce sens, elle permet une réflexion sur des enjeux dépassant l'exploitation agricole. Les résultats obtenus sur l'échelle socio-territoriale sont au-dessous de la moyenne (46/100) en raison des trois indicateurs qui présentent des notes planchers:

- Une démarche de qualité qui laisse à désirer

- Le manque du travail collectif

- La faible contribution à l'équilibre alimentaire

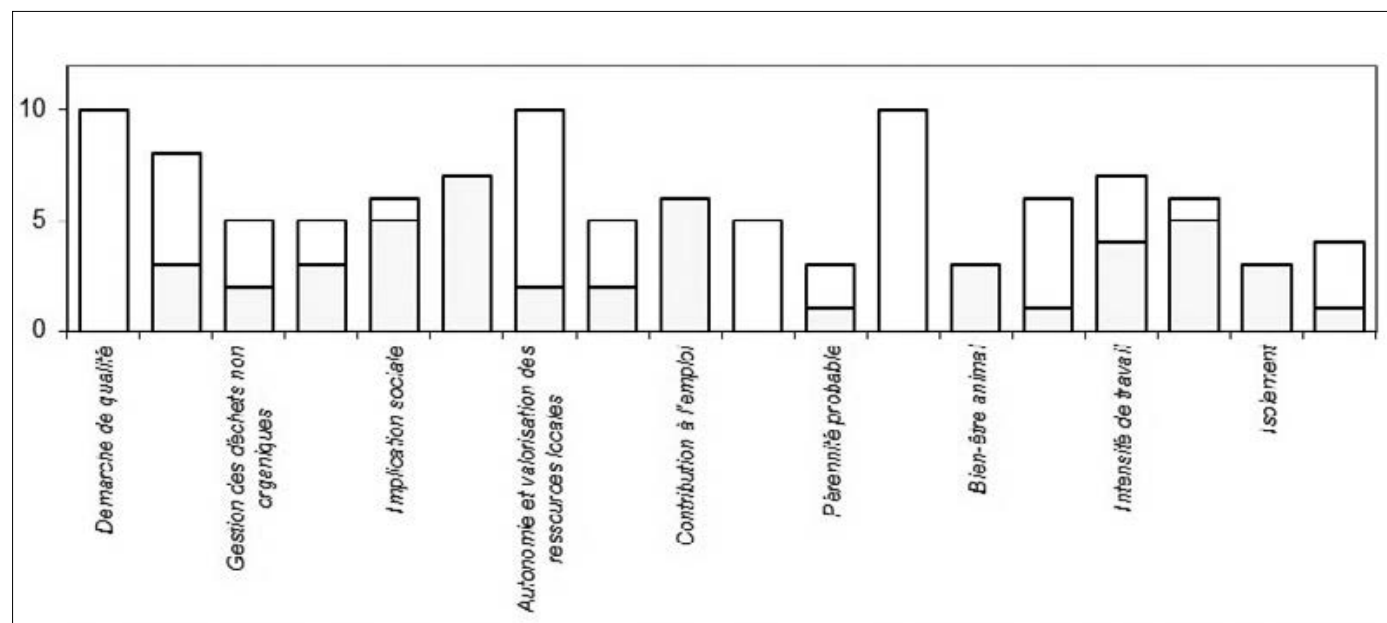

Figure 11 - Durabilité socio-territoriale.

Cette analyse montre que la démarche pour avoir une production agricole de qualité est nulle et s'explique par le fait que la destination finale de cette production est destinée à l'autoconsommation familiale ou bien au marché local (commercialisation ou transformation). Ce caractère familial ou local de l'issu de la production n'encourage pas les agriculteurs à élever le niveau de sa qualité.

Concernant la composante emploi et services, elle se caractérise par une absence de toute forme de participation au travail collectif chez les agriculteurs, vu l'absence de coopératives, la taille réduite des exploitations, le caractère saisonnier des cultures, le vieillissement des agriculteurs et surtout le manque d'intérêt de la population jeune envers l'activité agricole.

Les agriculteurs sont faiblement concernés par les activités subsidiaires liées au secteur primaire, notamment l'agrotourisme. Ce type d'activité n'a guerre dépassé des événements sporadiques (journées de dégustation, participation aux festivals locaux...). Concernant la valorisation par filières courtes, elle met en valeur le rapprochement entre les producteurs et les consommateurs dans les marchés locaux, en favorisant ainsi, une dynamique territoriale d'une part, et en diminuant les coûts de transports, d'une autre part.

En plus, ces agriculteurs ne voient pas l'utilité des sessions de vulgarisations ou de forma- 
tion organisées par les organismes techniques étatiques (CRDA, AVFA, Centres de formations agricoles ou de recherches...). Leurs techniques de gestion se basent sur leurs conditions socio-économiques ou sur leurs savoir faire ancestral.

b) Echelle de durabilité agro-écologique

L'échelle de durabilité agro-écologique analyse l'organisation du système technique avec le tandem valorisation du milieu et coût écologique minimum. Cette échelle rassemble des indicateurs illustrant la capacité des exploitations à être plus ou moins autonomes par rapport à l'utilisation des énergies et des matières non renouvelables relativement polluantes. Les indicateurs de cette échelle forment trois composantes d'importance égale: la diversité des productions, l'organisation de l'espace et les pratiques agricoles.

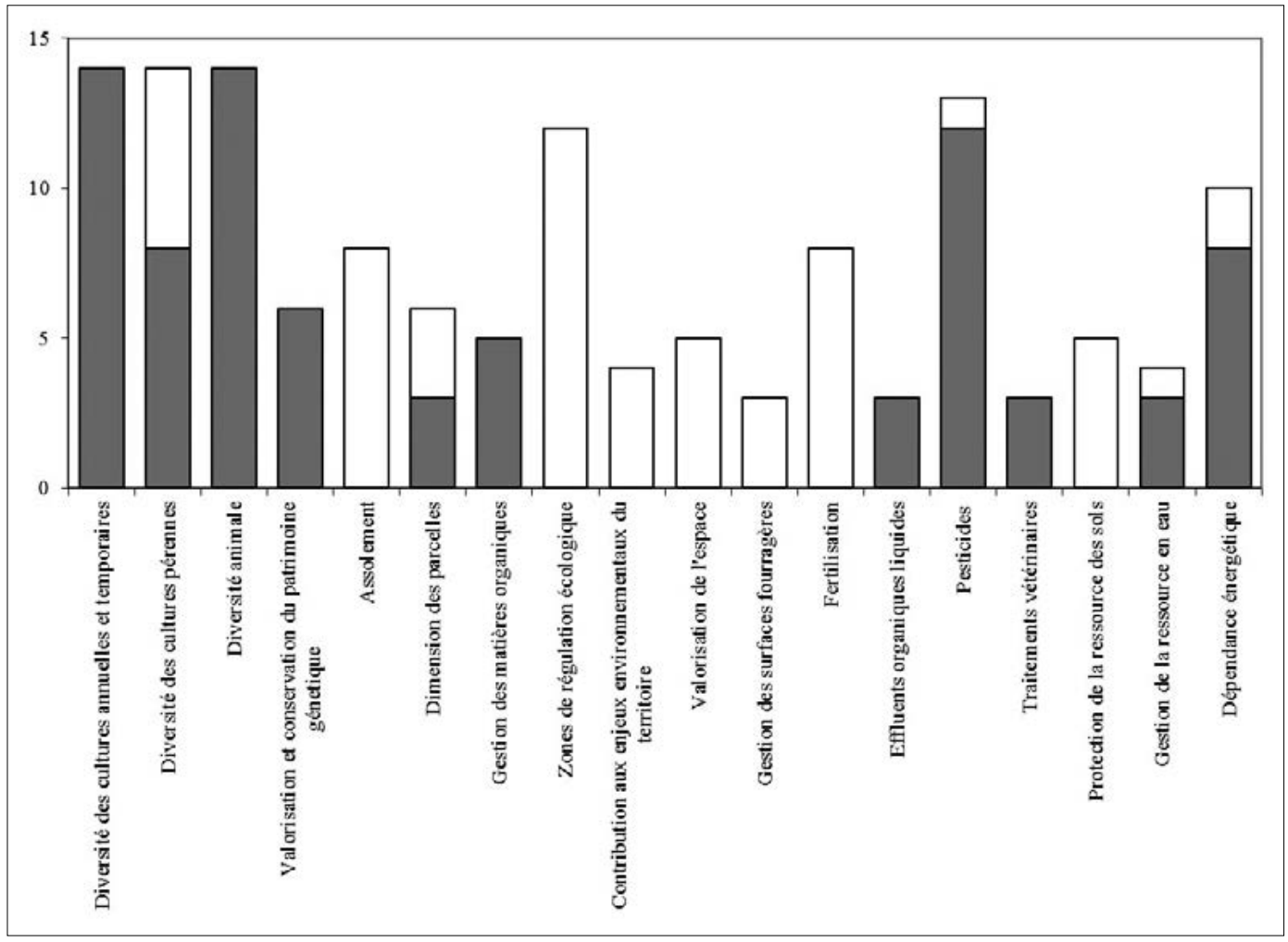

Figure 12 - Durabilité agro-écologique.

Les indicateurs obtenus relatifs à cette échelle sont les plus élevés et les mieux regroupés par rapport aux autres échelles. Dans les exploitations enquêtées, le score de diversité des cultures annuelles et temporaires est plafonné. Ces cultures annuelles se présentent essentiellement par les cultures maraîchères, favorisant la biodiversité domestique végétale, la cohérence technique et la gestion de la fertilité des sols à long terme, en les protégeant contre l'érosion. Toute- fois, l'absence des légumineuses de l'assolement indique un manque de complémentarité et de diversification en termes de spéculations agricoles.

Encore faut-il rappeler que les exploitations ont généralement une vocation oléicole avec quelques parcelles qui sont en mode de polyculture (cultures annuelles intercalaires entre les oliviers). La notion de surface assolable ne s'applique pas dans la mesure où ces cultures 
pérennes ne sont pas réensemencées chaque année.

On note l'absence des zones de régulation écologiques, et ce, malgré l'importance des ces espaces dans la protection de la biodiversité. Cependant, les agriculteurs pratiquent davantage la fertilisation organique plutôt que les engrais chimiques, non seulement pour une meilleure conservation de l'environnement, mais aussi, pour l'obtention de meilleurs rendements. Dans ce sens, ils utilisent des traitements phytosanitaires avec discernement et précaution en se dirigeant progressivement vers une agriculture biologique.

La majorité des agriculteurs ne prévoient pas d'aménagements antiérosifs. Chez quelques uns ceci se présente sous forme de plantations d'arbres ou d'arbustes, ou bien encore avec l'installation des systèmes de mise en défens.

\section{c) L'échelle de durabilité économique}

Cette dernière échelle, dont les indicateurs résultent des orientations techniques et fi- nancières du système de production, analyse les résultats économiques. En effet la pérennité d'un système de production dépend, non seulement de sa viabilité économique, mais aussi de son indépendance économique, de sa transmissibilité et de son efficience.

La viabilité économique des exploitations aussi bien à court qu'à moyen terme est naturellement une condition élémentaire de leur durabilité. Cet indicateur nous permet de mesurer la faisabilité économique des exploitations étudiées qui ont montré généralement une faible viabilité, liée entre autres à l'aspect financier.

Malgré les efforts déployés par les exploitants, en essayant de diversifier leurs productions et activités, le taux de spécialisation demeure faible et cela est à cause de la faible productivité des exploitations. L'autonomie financière, la sensibilité aux aides et la transmissibilité sont trois indicateurs plafonnés, favorisant la durabilité des exploitations irriguées dans la zone d'étude.

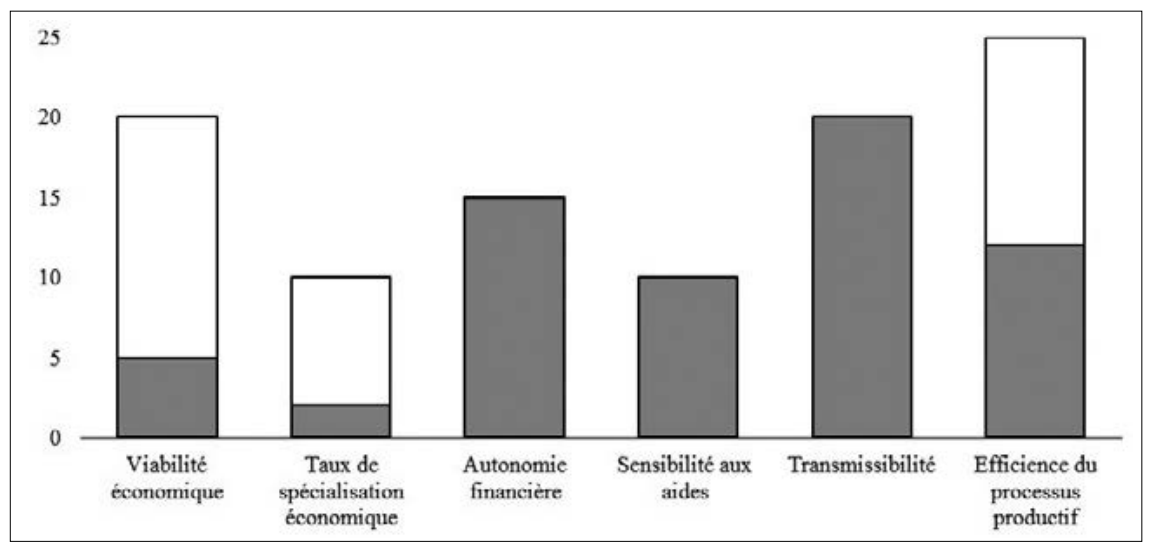

Figure 13 -

Durabilité économique.

\section{Conclusion et perspectives}

En l'absence des ressources naturelles renouvelables et vue les changements qui sont en interaction étroite et dont les effets sont reliés (changements climatiques, sécheresse, désertification, dégradation des sols cultivables diminution des ressources en eau, changement des habitudes humaines vers la consommation, extension de l'urbanisme, tendance vers les activités de service, exode rural...). L'agriculture en irrigué constitue une activité importante dans le système agraire existant, favorisant une bonne dynamique territoriale.

Toutefois, les résultats indiquent que la durabilité des exploitations en périmètre irrigué est 
limitée par l'échelle socio-territoriale. Ceci est du à l'absence de coopératives qui empêche les agriculteurs de créer de la valeur ajoutée à leur produits agricole, avec une démarche de qualité défaillante et des activités subsidiaires liées au secteur primaire très rares et sporadiques. Ceci se reflète aussi dans le manque de communication patent entre les organismes techniques étatiques (CRDA, AVFA, Centres de formations agricoles ou de recherches...) et les agriculteurs lors des planifications des sessions de vulgarisations ou de formation.

D'autre part, cette durabilité est soutenue par l'échelle agro-écologique, avec une bonne diversité des cultures (polyculture à base d'oléiculture, culture maraichères, céréaliculture...). Dans cet aspect les agriculteurs pratiquent davantage la fertilisation organique plutôt que les engrais chimiques, pour améliorer la fertilité du sol et diminuer les risques d'érosion, en se dirigeant progressivement vers une agriculture biologique.

Pour l'échelle économique les exploitants se caractérisent par leur autonomie financière émanant principalement des aides financières reçues de la part de la diaspora qui se trouve à l'étranger. Ils profitent aussi des subventions de l'Etat accordées à l'encouragement de l'agriculture en irriguée (Programmes d'économie d'eau, exonérations fiscale et douanières..). Le seul bémol c'est le tandem formé par la viabilité économique et le taux de spécialisation, qui demeure faible à cause de l'abandon de l'activité agricole, le manque de relève générationnelle, l'éloignement des exploitations des habitations, le caractère saisonnier des cultures, la faible productivité de plusieurs exploitations et le manque de formation des agriculteurs.

Quoi qu'il en soit, ce travail nous a permis de montrer qu'il s'agit d'un nouveau concept d'évaluation des exploitations agricoles dans les régions arides tunisiennes. En les enquêtant, cette étude a pour but de sensibiliser les agriculteurs à la notion de durabilité et à la nécessité de mieux prendre en compte la protection des milieux naturels dans ces régions difficiles. Cette méthode permettrait à ces agriculteurs de comprendre que la pérennité relève étroitement des trois piliers de l'agriculture durable, étant la protection de l'environnement, l'insertion dans son territoire économique et social et la performance économique.

De ce fait, le recours à des solutions qui favorisent une meilleure gestion de ces systèmes de production fragiles serait important pour contribuer à leur durabilité et à la viabilité des exploitations agricoles, tout en assurant un développement durable sur le plan économique, environnemental et social de l'agriculture irriguée.

En l'occurrence, pour assurer un développement durable et accroître la production dans cette région, la mise en œuvre d'un programme de sensibilisation et de formation des agriculteurs, à des modes de production adaptés aux conditions locales et plus économiques, notamment, au choix de cultures rentables avec une utilisation conservatrice et optimum des ressources en eau, devient impérative.

\section{Références bibliographiques}

Abdenour B., 2008. Essai d'adaptation de la méthode des indicateurs de durabilité des exploitations agricoles (IDEA) au contexte de l'élevage bovin laitier de la zone semi aride de Sétif. Alger: Institut National Agronomique El-Harrach, Alger.

APIA, 2014. Rapport annuel 2014: L'agriculture tunisienne, les atouts de la Tunisie. Tunisie. APIA. Tunis.

Apollin F., Eberhart C., 2012. Méthode de diagnostic d'un systeme irrigué pour le renforcement de la prise en charge de sa gestion par une association d'irrigants. Ministère de l'Agriculture, du Développement Rural et des Ressources Naturelles, Haiti.

Barbier J.M., Lopez-Ridaura S., 2010. Evaluation de la durabilité des systèmes de production agricoles: limites des démarches normatives et voies d'amélioration. Juin 2010, Montpellier: CIRAD-INRA-SUPAGRO.

Bonneviale J.R., Jussiau R., Marshall E., 1989. Approche globale de l'exploitation agricole. Comprendre le fonctionnement de l'exploitation agricole : une méthode pour la formation et le développement. Document INRAP Dijon, $329 \mathrm{p}$.

Debbarh A., Badraoui M., 2007. Irrigation et environnement au Maroc: situation actuelle et perspectives. Montpellier HAL-CIRAD. 
Feschet P., Bockstaller C., 2014. Méthodes d'évaluation multicritère des systèmes agricoles et $\mathrm{ACV}$ sociale, quelle complémentarité ? UMR LAE Nancy-Colmar: INRA.

Ferchichi A., Massin P., Kharrat M., Farhat N., Guenther N., 2015. Référentiel du développement agricole durable en Tunisie. Ministère de l'agriculture, des ressources hydrauliques et de la pêche, Agence de la vulgarisation et de la formation agricoles (AVFA).

Ghozlane F., Yakhlef H., Allane M., Bouzida S., 2006. Evaluation de la Durabilité des Exploitations Bovines Laitières de la Wilaya de Tizi-Ouzou (Algerie). New Medit, 4/2006.

ITES, 2009. Problématique de l'eau dans le monde, dans la région méditerranéenne \& en Tunisie.

Laajimi A., Ben Nasr J., 2009. Appréciation et comparaison de la durabilité des exploitations agricoles biologiques et conventionnelles en Tunisie: Cas de l'oléiculture dans la région de Sfax. New Medit, $1 / 2009$.

Landais E., 1998. Agriculture Durable : les fondements d'un nouveau contrat social. Courrier de l'environnement, INRA, n. 33.
M'hamdi N., Aloulou R., Hedhly M., Ben Hammouda M., 2009. Evaluation de la durabilité des exploitations laitières tunisiennes par la méthode IDEA». Base, 13(2): 221-228.

ODS, 2015. Gouvernorat de Médenine en chiffres. Ministère du Développement, de l'Investissement, et de la Coopération Internationale. Office de Développement du Sud.

Sghaier M., Picouet M., 2000. Observatoire des relations population-environnement en milieu rural tunisien: pour une gestion durable des ressources naturelles. DYPEN II. Rapport scientifique. IRA/ IRD.

Viaux P., 1999. Une troisième voie en grande culture: environnement, qualité, rentabilité. Paris: Editions Agridécisions, p. 211.

Vilain L., 2003. La Méthode IDEA, indicateurs de durabilité des exploitations agricoles. Guide d'utilisation. Dijon: Educagri éditions, p. 151.

Yakhelf H., Far Z., Ghozlane F., Madani T., Marie M., 2008. Evaluation de la durabilité des systèmes agropastoraux bovins dans le contexte de la zone semi-aride de Sétif (Algérie). New Medit, n. $4 / 2008$. 